\title{
GFDM 5G Cellular Network using MIMO System
}

\author{
Disha Kusumakar \\ MTech Student, ECE \\ LNCT, Indore
}

\author{
Deepak Pancholi \\ Assistant Professor \\ LNCT, Indore
}

\author{
Vikas Tiwari, PhD \\ Associate Professor, DOE \\ SVITS, SVV, Indore
}

\begin{abstract}
This paper presents the generalized frequency division multiplexing a GFDM system with MIMO system which is much more diverse application requirements. The $5 \mathrm{G}$ physical layer will support applications with different requirements, such as high data rate, ultra low power consumption and low latency. Recently, there is significant interest in the design and performance of new waveforms for 5G. One of the most important candidates is the Generalized Frequency Division Multiplexing (GFDM) waveform. Multiple-input multipleoutput (MIMO)-friendliness is a key ability for a physical layer scheme to satisfactorily match the requirements of future. MIMO-GFDM schemes are provided through the proposed framework and their bit error ratio performances, computational complexities, and spectral efficiencies are analyzed. It has been demonstrated that the Intersymbol interference and noise will be reduce with the better performance.
\end{abstract}

\section{Keywords}

5G Wireless Network, GFDM, MIMO, OFDM.

\section{INTRODUCTION}

Mobile communication has become an essential tool for the modern society. The first generation of cellular systems provided basic, yet innovative, voice transmission. Communication started to become personal rather than being connected to fixed locations. The second generation has digitalized the voice in order to increase system capacity, battery life of devices and Quality of Service (QoS). It also introduced the Short Message Service, which revolutionized the way people communicate [1]. The third generation enabled mobile Internet access and data rates not too far behind of wired solutions of that time. The advent of smart phones with large storage and processing capabilities equipped with high definition screen and cameras, in combination with social networks that turned users from media consumers into content providers, has pushed the fourth generation towards even higher throughput. Starting with the second generation, the evolution of the mobile communication has focused on increasing the throughput. However, the scenarios foreseen for future fifth generation (5G) networks have requirements that clearly go beyond higher data rates. In this paper we show that space-time coding (STC) can be effectively combined with GFDM for achieving transmit and receive diversity Fourth generation $(4 \mathrm{G})$ cellular systems have been optimized to provide high data rates and reliable coverage to mobile users in very dense areas [2]. The next generation 5G cellular systems will face much more diverse application requirements.

\subsection{OFDM}

At present, Orthogonal Frequency Division Multiplexing (OFDM) is a widely adopted solution mainly because of its robustness against multipath channels and easy implementation based on Fast Fourier Transform (FFT) algorithms. But the application scenarios predicted for $5 \mathrm{G}$ networks present challenges which OFDM can only address in a limited way. Furthermore, all major synchronization algorithms developed for OFDM can be adapted for GFDM. Of course, multiple input multipleoutput (MIMO) will be a key feature in $5 \mathrm{G}$ networks. The OFDM is the core of the physical layer of fourth generation $(4 \mathrm{G})$ wireless networks and fulfills the requirements and challenges of $4 \mathrm{G}$ scenarios. Despite of its proven advantages, OFDM has some shortcomings that make it difficult to address the scenarios foreseen for future $5 \mathrm{G}$ wireless networks. In OFDM, every symbol requires a cyclic prefix $(\mathrm{CP})$, which depends on the tap length of the channel. The insertion of $\mathrm{CP}$ reduces the spectral efficiency and prevents obtaining a low latency by shortening the symbols. Furthermore, OFDM is very sensitive to time and frequency synchronization errors and has high out-of-band (OOB) emission due to rectangular pulse shaping. Thus, OFDM can fulfill the requirements of $5 \mathrm{G}$ wireless networks in a limited way. In recent years, several waveform proposals have been presented to overcome the above limitations of OFDM. These proposals can be categorized into two main classes: cyclicly-prefixed OFDM (CP-OFDM)based and non-CPOFDM- based. The proposals in the first class, such as filtered OFDM (f-OFDM), and windowed OFDM (WOFDM), are the attempts to resolve the aforementioned problems by keeping the orthogonality. The proposals in the second class initially dismiss orthogonality to obtain better temporal and spectral characteristics, thus, causes a major paradigm shift in the context of waveform design, which may yield some backward compatibility issues. For avoiding this issue we can use F-OFDM

\subsection{FOFDM}

it reduces complexity of ofdm.it can be combined with multiantenna system as filter length is less than FBMC. it intentionally give orthogonality between consequetive symbols allowing min, no. of guard tones. OFDM makes it easier to place narrow band signals in small frequency space. Possibility to incorporate other waveforms such as GFDM, FBMC, UFMC etc.

\subsection{FBMC}

Filter Bank Multicarrier each subcarrier is filtered individually. It uses the very narrow band filter with long time length. Due to the use of filter for each subcarrier, OOB emissions are greatly reduced. In FBMC, first prototype filter is to be designed. After that filters are designed for each subcarrier based on the prototype filter by frequency shifting. All the filters together are called filter bank. The main difference between OFDM and FBMC is OFDM uses one rectangular filter for all subcarriers whereas FBMC uses one filter for each subcarrier. FBMC has high spectral efficiency when compared to OFDM because cyclic prefix is not used in FBMC. 


\subsection{UFMC}

UFMC combines the advantages of OFDM and filter bank in FBMC. In UFMC, first the total bandwidth is divided into sub-bands. Each sub-band has some subcarriers UFMC has more spectral efficiency compared to OFDM. There is no cyclic prefix insertion like in OFDM. There is no repetition of the same bits, therefore it utilizes all the allocated spectrum efficiently. UFMC has less side lobes than OFDM. As side lobes decreases the interference on adjacent subcarriers also decreased. In OFDM, the signal consists of a large number of independently modulated subcarriers which can give a large PAPR when they are added in phase. In UFMC, total bandwidth is divided into sub-bands. As the probability of number of subcarriers adding up in phase is less in UFMC, the maximum power decreases. Hence PAPR is low for UFMC when compared with ofdm.

\section{GFDM:}

It is a generalized form of OFDM, which keeps most of the advantageous properties of OFDM while addressing its limitations and discuss that the limitations due to the carrier aggregation in an OFDM system is eliminated by using GFDM system, since it provides a very low out-of-band radiation. Moreover, GFDM uses circular filtering instead of linear filtering used in OFDM, which eliminate the prototype filter transient intervals and hence the latency. Because of this, it can provide for low latency applications like internet of things (IoT) and M2M. It is also robust to loss of orthogonality and limits the intercarrier interference (ICI), by filtering the subcarriers using a well-designed prototype filter.

GFDM MIMO system: the performance of MIMO-GFDM systems can be enhanced accordingly. In addition to this iterative detection algorithm, we also propose a new nulling filter design based on a modified error detection criterion, which is capable of removing the residual interference. Multiple-input multiple-output (MIMO)-friendliness is a key ability for a physical layer scheme to satisfactorily match the foreseen requirements of future wireless networks. Since GFDM is a generalized form of OFDM, one can expect that its combination with MIMO is feasible.This was shown for space-time coding (STC) technique and spatial multiplexing and iterative MIMO decoders have been evaluated. In separate detection and demodulation (SDD) with minimum mean squared error (MMSE) detector and zero-forcing (ZF) GFDM demodulation was proposed an equivalent MIMO-GFDM channel model, which combines GFDM modulation and MIMO channel, has been presented and was analyzed along with the CP-OFDM-based counterparts. Nevertheless, when spatial multiplexing is employed with GFDM, inter-antenna interference (IAI) is added to inherent self-interference of GFDM and makes the receiver design challenging. The continuing demand for higher data rates motivates the researchers to seek spectrally efficient new modulation schemes. Spatial modulation (SM) is a MIMO transmission method, which considers the transmit antennas as spatial constellation points to carry additional information bits [18]. In SM, at each time interval, a single transmit antenna is activated by the input bit sequences and other antennas remainsilent. For SM multicarrier schemes, only one transmit antenna is activated at any subcarrier. As a result, activating single transmit antenna at a time or subcarrier eliminates IAI and reduces the receiver complexity. Moreover, SM techniques are more robust to channel imperfections and enhance the error performance. SM-based modulation schemes have recently received a great deal of interest due to their attractive advantages over classical MIMO systems.
Space-shift keying (SSK) is a special case of SM, where only active transmit antenna indices are used to convey information. In SSK, phase shift keying (PSK)/quadrature amplitude modulation (QAM) symbols are not used and active transmit antenna transmits a fixed non-data bearing signal. Space-time shift keying (STSK) is another SM-based modulation scheme, where both the indices of multiple preassigned dispersion matrices and signal constellation points are used to convey information. Additionally, the concept of $\mathrm{SM}$ is extended to include both the space and time dimensions. Quadrature SM (QSM) expands the spatial constellation symbols to in-phase and quadrature components, and doubles the number of spatially carried bits with respect to a conventional SM system. In QSM, signal constellation symbol is divided into its real and imaginary parts and their corresponding transmit antennas are determined by the input bit sequences in a separate fashion. It is demonstrated that QSM achieves the same error performance and spectral efficiency using $3 \mathrm{~dB}$ less signal power with respect to its SM counterpart without increasing the receiver complexity.

\section{REVIEW OF GFDM}

In 2014, Nicola Mikhailov et al. have been proposed waveform and highlight relevant features of GFDM. After introducing the principles of GFDM, this paper contributes to the following areas: (i) means for engineering the waveform's spectral properties, (ii) analytical analysis of symbol error performance over different channel models, (iii) concepts for MIMO-GFDM to achieve diversity, (iv) preamble-based synchronization that preserves the excellent spectral properties of the waveform, (v) bit error rate performance for channel coded GFDM transmission using iterative receivers, (vi) relevant application scenarios and suitable GFDM parameterizations, (vii) GFDM proof-of-concept and implementation aspects of the prototype using hardware platforms available today. In summary, the flexible nature of GFDM makes this waveform a suitable candidate for future $5 \mathrm{G}$ networks [1].

In 2015, presented an enabler for flexible waveform configuration, named as filtered-OFDM (f-OFDM). With the conventional OFDM, a unified numerology is applied across the bandwidth provided, balancing among the channel characteristics and the service requirements, and the spectrum efficiency is limited by the compromise we made. In contrast, with f-OFDM, the assigned bandwidth is split up into several sub bands, and different types of services are accommodated in different sub bands with the most suitable waveform and numerology, leading to an improved spectrum utilization. After outlining the general framework of f-OFDM, several important design aspects are also discussed, including filter design and guard tone arrangement. In addition, an extensive comparison among the existing $5 \mathrm{G}$ waveform candidates is also included to illustrate the advantages of f-OFDM. Our simulations indicate that, in a specific scenario with four distinct types of services, f-OFDM provides up to $46 \%$ of throughput gains over the conventional OFDM scheme [2].

In 2017, the advantages of non-CP-OFDM-based waveforms and the IM concept, we present a framework, which integrates GFDM with space and frequency IM schemes to provide flexible and advanced novel RATs for future wireless networks. Several MIMO-GFDM schemes are provided through the proposed framework and their bit error ratio (BER) performances, computational complexities and spectral efficiencies are analyzed. Based on the obtained results, a guideline for selecting the proper MIMO-GFDM scheme considering target performance criterion is given. It has been 
demonstrated that the proposed framework has a strong potential to engineer the space-frequency structure according to channel conditions and use-cases, and it provides a great flexibility that can be easily tuned to address the required performance criterion [3]

In 2018, Muhammad Asmi Islam and Aamir Habib, implement transmit diversity to GFDM using standard OSTBC for higher number of antennas and compare the results with OFDM. We also present the ML decoding equations for OSTBC of eight transmit antennas, to compete the requirements of $5 \mathrm{G}$, a number of candidate waveforms were proposed. Next generation cellular system will face more diverse application requirements. GFDM was also a candidate waveform, which in research shows a number of benefits over others candidate waveforms. This waveform is basically built on filtered multi-carrier methodology that offers extra flexibility, which is a key requirement of upcoming cellular applications. To cope with the modern requirements, every candidate waveform needs to perform better in MIMO environment [4].

\section{CONCLUSION}

In this paper, studied and identified the GFDM technique, a strong candidate for the physical layer of future generation cellular communication systems. Apart from giving better BER performance, the GFDM MIMO system addresses some drawbacks of the OFDM system, like reduced out-of-band emission by the circularly shaping the sub-carrier pulses. Numerous issues still need to be resolved, like higher computational complexity and delays as compared to OFDM because of the need for individual subcarrier processing in GFDM. However, our study delineates that GFDM has the capability to satisfy the necessities of the next generation mobile wireless systems.

\section{REFERENCES}

[1] Nicola Mikhailov, Maximilian Matthew, Ivan Samos Gaspar, Ayona Navarro Clareville, Luciano Leonel
Mendes,Andreas Festag and Gerhard Fettweis, "Generalized Frequency Division Multiplexing for 5th Generation Cellular Networks", IEEE, 2014. 0090-6778 2013 10.1109/TCOMM.2014.2345566

[2] Xi Zhang, Ming Jia, Lei Chen, Jianglei Ma, Jing Qiu, "Filtered-OFDM - Enabler for Flexible Waveform in the 5th Generation Cellular Networks", IEEE, Vol., pp, 2015. 978-1-4799-5952-5/15/\$31.00 @2015

[3] Enver Hamiti, Fatlum Sallahu "Spectrum Comparison between GFDM, OFDM andGFDM Behavior in a Noise and Fading Channel" Preliminary Communication vol 6 . 2015

[4] Vitthal Lamani and Dr. Prerana Gupta Poddar "Generalized Frequency Division Multiplexing for 5G Cellular Systems: A Tutorial Paper" AKGEC International Journal of Technology, Vol. 8, No. 12015

[5] Ersin Ozturk, Ertugrul Basar, Hakan Ali Cirpan Faculty of Electrical and Electronics Engineering (2016), "Spatial Modulation GFDM: A Low Complexity MIMOGFDM System For 5G Wireless Networks". IEEE

[6] ERS'IN ÖZTÜRK, ERTUGRUL BASAR, and HAKAN AL'I CIRPAN1, "Generalized Frequency Division Multiplexing with Flexible Index Modulation" (Member, IEEE) 2017

[7] Aitor Lizeaga,Pedro M. Rodríguez, Iñaki Val,and Mikel Mendicute "Evaluation of $5 \mathrm{G}$ Modulation Candidates WCP-COQAM, GFDM-OQAM, and FBMC-OQAM inLow-Band Highly Dispersive Wireless Channels" Hindawi Journal of Computer Networks and Communications Volume 2017, Article ID 2398701,

[8] JIE ZHONG1, GAOJIE CHEN, JUQUAN MAO, SHUPING DANG, PEI XIAO, "Iterative Frequency Domain Equalization for MIMO-GFDM Systems", 2018 